\title{
MAPPING CYLINDER NEIGHBORHOODS OF ONE-COMPLEXES IN FOUR-SPACE
}

\author{
BY \\ J. L. BRYANT( $\left.{ }^{(}\right)$AND R. C. LACHER( $\left.{ }^{2}\right)$
}

\begin{abstract}
We prove the following theorem: If $K$ is a 1-complex topologically embedded in $S^{4}$, and if $K$ has mapping cylinder neighborhoods in $S^{4}$ at almost all of its points, then $K$ is tame. The proof uses engulfing and the theory of proper, one-acyclic mappings of 3-manifolds onto the real line.
\end{abstract}

Suppose $M^{m}$ is a (topological) manifold (without boundary) in the manifold $N^{n}, x \in M^{m}$. We say that $M^{m}$ has a mapping cylinder neighborhood in $N^{n}$ at $x$ if there exist

(i) an open neighborhood $V$ of $x$ in $M^{m}$;

(ii) an open $(n-1)$-manifold $U$;

(iii) a proper mapping $\phi$ of $U$ onto $V$; and

(iv) a homeomorphism $\psi$ of $Z_{\phi}$ onto a neighborhood of $V$ in $N$ such that $\psi(v)=v$ for each $v \in V$.

The requirement that the image of $\psi$ be a neighborhood of $V$ forces $U$ to have two components when $m=n-1$ (and $V$ is small).

In the above definition, $Z_{\phi}$ denotes the mapping cylinder of $\phi$, with $U$ and $V$ identified as subsets of $Z_{\phi}$ as is customary. A proper map is one under which inverse images of compact sets are compact. We use $\boldsymbol{R}^{n}$ to denote euclidean $n$-space, $B^{n}$ is the closed unit ball centered at 0 in $R^{n}$, and $S^{n}=\operatorname{Bd} B^{n+1}$. The symbol " $\approx$ " means "is homeomorphic to".

It has been conjectured that an $m$-manifold $M^{m}$ is locally flat in an $n$-manifold $N^{n}$ if and only if $M^{m}$ has mapping cylinder neighborhoods in $N^{n}$ at each point. When $n \leqq 3$, this conjecture has been proved by Nicholson in [17]. The case $n=4$, $m=3$ is proved in [14]. The main purpose of the present paper is to prove the conjecture in case $n=4, m=1$ (see Corollary 4.2 below). The cases $n-m=2$, $n \geqq 4$ are false (because there are nonlocally flat piecewise linear embeddings). When $n \geqq 5$, the cases $n-m \neq 2$ remain open.

The truth of the above mapping cylinder conjecture in case $n=k+l, m=l-1$ implies the truth of the following well-known conjecture: If the $l$-fold suspension

Received by the editors December 21, 1970.

AMS 1970 subject classifications. Primary 55A99, 57A30, 57A35, 57A40, 57A50.

Key words and phrases. Topological embeddings of one-complexes, locally flat embeddings, locally tame embeddings, mapping cylinder neighborhoods, UV properties, engulfing.

(1) Partially supported by grant NSF GP-19964.

(2) Alfred P. Sloan Research Fellow, partially supported by grant NSF GP-19964.

Copyright (C) 1972, American Mathematical Society 
of a homology $k$-sphere $H^{k}$ is a manifold, then $H^{k}$ is simply connected. Thus, for $n \geqq 5$, the cases $n-m=1$ might seem the more tractable of the mapping cylinder conjectures. See [7] for the latest on the homology sphere conjecture.

\section{Acyclicity of certain maps.}

Definition. Let $Z$ be a space, $Y$ a closed subspace, $G$ an abelian group. We say that $Z$ is $\operatorname{lc}^{k}(G) \bmod Y$ if, for any point $y \in Y$ and any neighborhood $U$ of $y$ in $Z$, there exists a neighborhood $V$ of $y$ in $U$ such that the inclusion-induced map $\tilde{H}_{i}(V-Y ; G) \rightarrow \tilde{H}_{i}(U-Y ; G)$ is zero (on reduced singular homology) for $0 \leqq i \leqq k$.

The following result is well known. ( $Z$ is the group of integers.)

THEOREM 1.1. Suppose that $M^{m}$ is an m-manifold topologically embedded in the interior of the n-manifold $N^{n}$. Then $N^{n}$ is $\operatorname{lc}^{n-m-2}(Z) \bmod M^{m}$.

Proof. Let $x \in M$, and let $U$ be an open $n$-cell neighborhood of $x$ in $N$. Consider the reduced homology sequence of the pair $(U, U-M)$ :

$$
\cdots \rightarrow H_{i+1}(U, U-M) \rightarrow \tilde{H}_{i}(U-M) \rightarrow \tilde{H}_{i}(U) \rightarrow \cdots .
$$

We have $\widetilde{H}_{i}(U)=0$ for all $i$ and $H_{i+1}(U, U-M) \simeq H_{c}^{n-i-1}(U \cap M)=0$ for $n-i-1$ $>m$. Therefore $\tilde{H}_{i}(U-M)=0$ for $i \leqq n-m-2$.

Definition. A compact set $X$ in the ANR $M$ is said to have property $\mathrm{uv}^{k}(G)$ (or be strongly $k$-acyclic over $G$ ) if for any neighborhood $U$ of $X$ in $M$ there exists a neighborhood $V$ of $X$ in $U$ such that $\tilde{H}_{i}(V ; G) \rightarrow \tilde{H}_{i}(U ; G)$ is zero for $0 \leqq i \leqq k$.

COROLlaRY 1.2. Suppose that $f: U^{n-1} \rightarrow V^{m}$ is a proper, surjective map between manifolds. If $Z_{f}$ embeds (locally) in euclidean $n$-space then $f^{-1}(y)$ has property $u^{n-m-2}(Z)$ for each $y \in V^{m}$.

Proof. It follows immediately from (1.1) that $Z_{f}$ is $\operatorname{lc}^{n-m-2}(Z) \bmod V$. The result follows from Theorem 2.2 of [13].

2. One-acyclic maps of 3-manifolds onto $R$. Let $Z_{*}$ be either the integers or the integers modulo two, $\boldsymbol{R}$ the real line.

THEOREM 2.1. Let $W^{3}$ be an open 3-manifold, and suppose that $f: W^{3} \rightarrow \boldsymbol{R}$ is a proper, surjective map. Suppose further that $f^{-1}(t)$ has property $\mathrm{uv}^{1}\left(Z_{*}\right)$ for each $t \in \boldsymbol{R}$. Then there exists a locally finite subset $F$ of $\boldsymbol{R}$ such that if $J$ is an open interval of $\boldsymbol{R}$ which contains no point of $F$ then $f^{-1}(J) \approx S^{2} \times \boldsymbol{R}$.

Before proving (2.1) we need a definition and a preliminary result.

Definition. A compact set $X$ in the ANR $M$ is said to have property 1-UV if each neighborhood $U$ of $X$ in $M$ contains a neighborhood $V$ of $X$ such that every loop in $V$ is null-homotopic in $U$.

THEOREM 2.2. Let $f$ be a proper, monotone map of the open 3-manifold $W^{3}$ onto $\boldsymbol{R}$. Suppose that, for each $t \in \boldsymbol{R}, f^{-1}(t)$ has a neighborhood which contains no fake cubes and $f^{-1}(t)$ has property 1-UV. Then $W^{3} \approx S^{2} \times \boldsymbol{R}$. 
Proof. Since $f \mid f^{-1}(U)$ induces an isomorphism $\pi_{1}\left(f^{-1}(U)\right) \rightarrow \pi_{1}(U)$ for any connected open set $U \subset \boldsymbol{R}$ (see [1] or [12]) we have that $f^{-1}(J)$ is simply connected for any open interval $J \subset \boldsymbol{R}$, and, moreover, $f^{-1}(J)$ has two simply connected ends.

Choose $J$ such that $f^{-1}(J)$ contains no fake cubes. Applying [9], we see that either end of $f^{-1}(J)$ has a collar neighborhood; thus, there exists an embedding

$$
h: S^{2} \times((-\infty,-1) \cup(1,+\infty)) \rightarrow f^{-1}(J)
$$

such that the complement of the image of $h$ is compact. Let $W_{-}=h\left(S^{2} \times(-\infty,-2]\right)$, $W_{+}=h\left(S^{2} \times[2,+\infty)\right)$, and $W_{0}=f^{-1}(J)-W_{-}^{\circ}-W_{+}^{\circ} . W_{0}$ is a compact simply connected 3-manifold whose boundary is the union of two 2-spheres. Moreover, $W_{0}$ contains no fake cubes. Such a 3 -manifold must be homeomorphic to $S^{2} \times I$; for, by boring a hole from one boundary component to the other, we can write $W_{0}$ as the union of a 3-cell and a homotopy 3-cell meeting along an annulus common to their boundaries. Thus, $f^{-1}(J) \approx S^{2} \times \boldsymbol{R}$.

Now, let $\cdots t_{i-1}<t_{i}<t_{i+1}<\cdots$ be a locally finite set in $R$ such that $f^{-1}\left(t_{i}, t_{i+2}\right)$ $\approx S^{2} \times \boldsymbol{R}$ for each $i$. We can engulf any compact set in $W$ with $f^{-1}\left(t_{-1}, t_{+1}\right)$ using the structure of $f^{-1}\left(t_{i}, t_{i+2}\right)$ to pull $f^{-1}\left(t_{-1}, t_{+1}\right)$ along. Thus, $W$ contains no fake cubes, and the result follows from the argument in the preceding paragraph.

Proof of (2.1). Let $f: W^{3} \rightarrow \boldsymbol{R}$ be given as in the hypothesis of (2.1). Define

$$
\begin{aligned}
F^{\prime} & =\left\{t \in \boldsymbol{R} \mid f^{-1}(t) \text { does not have property 1-UV }\right\}, \\
F^{\prime \prime} & =\left\{t \in \boldsymbol{R} \mid \text { every neighborhood of } f^{-1}(t) \text { contains a fake cube }\right\}, \\
F & =F^{\prime} \cup F^{\prime \prime}
\end{aligned}
$$

By (2.2), we need only show that $F$ is locally finite. A theorem of Kneser [11] shows that $F^{\prime \prime}$ is locally finite. The local finiteness of $F^{\prime}$ follows from work of McMillan [15] (see also [16]) and Wright [18]. Let $F_{0}^{\prime}=\left\{t \in \boldsymbol{R} \mid f^{-1}(t)\right.$ does not have arbitrarily small neighborhoods with free fundamental group $\}$. It follows from Theorem 2 of [15] that $F_{0}^{\prime}$ is locally finite (for otherwise a compact submanifold of $W$ has nonfinitely generated fundamental group by Grushko's theorem). But the arguments [18, Theorem 1] show that $F_{0}^{\prime} \supset F^{\prime}$. (If $t \notin F_{0}^{\prime}$, then $f^{-1}(t)$ has small neighborhoods $V \subset U$, where $H_{1}\left(V ; Z_{2}\right)=0$ and $\pi_{1}(U)$ is free. Let $S_{0} G=G$, $S_{n+1} G=$ subgroup of $S_{n} G$ generated by all squares of elements of $S_{n} G$, and $S_{\omega} G$ $=\bigcap_{n=1}^{\infty} S_{n} G$. It follows that $S_{\omega} \pi_{1}(V)=\pi_{1}(V)$ while $S_{\omega} \pi_{1}(U)=\{1\}$, so that every homomorphism $\pi_{1}(V) \rightarrow \pi_{1}(U)$ is trivial.)

REMARK. Examples show that $F$ in (2.1) may well be nonvoid. Using connected sum, stick homology spheres onto $S^{2} \times \boldsymbol{R}$ to form $W^{3}$; the map $f$ is to shrink spines of the homology spheres to points and then project to $\boldsymbol{R}$.

\section{A special case of the main theorem.}

THEOREM 3.1. Suppose that $\phi: S^{2} \times \boldsymbol{R} \rightarrow \boldsymbol{R}$ is a proper, surjective map such that $\phi^{-1}(J) \approx S^{2} \times \boldsymbol{R}$ for any open interval $J \subset \boldsymbol{R}$. Suppose further that $\psi: Z_{\phi} \rightarrow \boldsymbol{R}^{4}$ is an embedding of $Z_{\phi}$ onto a neighborhood of $\psi(\boldsymbol{R})$ in $\boldsymbol{R}^{4}$. Then $\psi(\boldsymbol{R})$ is locally flat in $\boldsymbol{R}^{4}$. 
Throughout the remainder of this section we shall adhere to the following notation:

(a) $\phi: S^{2} \times R \rightarrow R$ and

(b) $\psi: Z_{\phi} \rightarrow R^{4}$

are as in the hypothesis of Theorem 3.1.

(c) $S_{0}=\psi\left(Z_{\phi}\right)$.

(d) For $0 \leqq t<1, S_{t}$ denotes the portion of $\psi\left(Z_{\phi}\right)$ between the levels $t$ and 1 , the range of $\phi$ being the 1-level of the mapping cylinder $Z_{\phi}$.

We may assume that $\psi\left(S^{2} \times \boldsymbol{R}\right)=\mathrm{Bd} S_{0}$ is locally flat in $\boldsymbol{R}^{4}$, since $S_{0}$ may be replaced by $S_{t}(t>0)$.

LEMMA 3.2. Given a finite subset $\left\{s_{1}, s_{2}, \ldots, s_{n}\right\}$ of $\boldsymbol{R}$ with $s_{1}<s_{2}<\cdots<s_{n}$, there exists an embedding $g: B^{3} \times[1, n]$ into $S_{0}$ such that $g^{-1}\left(\mathrm{Bd} S_{0}\right)=S^{2} \times[1, n]$ and $g(0, k)=\psi\left(s_{k}\right)$ for $k=1, \ldots, n$.

Proof. We first show how to find a 3-cell $B$ in $S_{0}$. Let $\alpha: S^{2} \times(-1,1) \rightarrow \phi^{-1}(-1,1)$ be a homeomorphism. Let $\Sigma_{t}$ be $\alpha\left(S^{2} \times\{t\}\right) \times\{t\}$, copied in $Z_{\phi}(0 \leqq t<1)$. Clearly $B=\psi\left(\bigcup_{0 \leqq t<1} \Sigma_{t} \cup\{1\}\right)$ is a 3-cell in $S_{0}$ that is locally flat in $S_{0}$ except possibly at $\psi(1)$. A result of Kirby [10] then implies that $B$ is locally flat. From the construction of $B$ it is also clear that $B$ meets $B d S_{0}$ "nicely".

Now, for $k=1, \ldots, n$, let $B_{k}$ be a 3-cell in $\psi\left(Z_{\phi \mid \phi^{-1}\left(s_{k-1}, s_{k+1}\right)}\right)$ as constructed above with $B_{i} \cap B_{j}=\varnothing$ if $i \neq j$ and $B_{k} \cap \psi(R)=\psi\left(s_{k}\right)$. Let $A_{k}$ be the annulus in $\mathrm{Bd} S_{0}$ bounded by $\mathrm{Bd} B_{k}$ and $\mathrm{Bd} B_{k+1}(k=1, \ldots, n-1)$. Then $B_{k} \cup A_{k} \cup B_{k+1}$ is a locally flat 3-sphere in $S_{0}$ that bounds a 4-cell $C_{k}$ in $S_{0}$ by the generalized Schoenflies Theorem [2]. Let $g_{k}: B^{3} \times[k, k+1] \rightarrow C_{k}$ be a homeomorphism that takes $B^{3} \times\{k\}$ onto $B_{k}$ and $B^{3} \times\{k+1\}$ onto $B_{k+1}$. Construct the $g_{k}$ 's inductively so that $g_{k}\left|B^{3} \times\{k+1\}=g_{k+1}\right| B^{3} \times\{k+1\} \quad(k=1, \ldots, n-1)$. Defining $g: B^{3} \times[1, n]$ $\rightarrow S_{0}$ by $g \mid B^{3} \times[k, k+1]=g_{k}$, we complete the proof of the lemma.

REMARK 1. It is clear that in the statement of Lemma 3.2 we could have replaced $S_{0}$ by $S_{t}$ for any $t \in[0,1)$.

REMARK 2. The proof of Theorem 3.1 could now be completed by appealing to the technique of [3] as explained in [4]. The reason this is so is that for $t$ sufficiently close to 1 and for suitably chosen $s_{1}<0<s_{2}<\cdots<s_{n-1}<1<s_{n}$, we can get a small push of $\psi([0,1])$ off of any 2-complex $K$ by first placing $g(\{0\} \times[1, n])$ and $K$ in general position. We have a complete, elementary proof, however, that uses a construction similar to one found in [6].

Lemma 3.3. Suppose that $\left\{s_{1}^{\prime}, \ldots, s_{m}^{\prime}\right\}$ and $\left\{s_{1}^{\prime \prime}, \ldots, s_{n}^{\prime \prime}\right\}$ are subsets of $\boldsymbol{R}$ with $s_{1}^{\prime}<\cdots<s_{m}^{\prime}, s_{1}^{\prime \prime}<\cdots<s_{n}^{\prime \prime}$, and $s_{i}^{\prime} \neq s_{j}^{\prime \prime}$ (for any $\left.i, j\right)$, that $t^{\prime}, t^{\prime \prime} \in[0,1)$, and that $g^{\prime}: B^{3} \times[1, m] \rightarrow S_{t^{\prime}}$ and $g^{\prime \prime}: B^{3} \times[1, n] \rightarrow S_{t^{\prime \prime}}$ are the associated embeddings obtained from Lemma 3.2. Let

$$
\left\{s_{1}, \ldots, s_{m+n}\right\}=\left\{s_{1}^{\prime}, \ldots, s_{m}^{\prime}\right\} \cup\left\{s_{1}^{\prime \prime}, \ldots, s_{n}^{\prime \prime}\right\}
$$


arranged so that $s_{1}<\cdots<s_{m+n}$. Then there exist $t \in[0,1)$ and an embedding $g: B^{3} \times[1, m+n] \rightarrow S_{t}$ such that

$$
\begin{aligned}
g\left(B^{3} \times\{k\}\right) & =g^{\prime}\left(B^{3} \times\{i\}\right) \cap S_{t} \text { if } s_{k}=s_{i}^{\prime}, \\
& =g^{\prime \prime}\left(B^{3} \times\{j\}\right) \cap S_{t} \text { if } s_{k}=s_{j}^{\prime \prime} .
\end{aligned}
$$

Proof. Observe that the 3-cell $B$ constructed at the beginning of the proof of Lemma 3.2 separates each $S_{t}$ in the same manner in which it separates $S_{0}$. Furthermore, given $g^{\prime}: B^{3} \times[1, m] \rightarrow S_{t^{\prime}}$ and $g^{\prime \prime}: B^{3} \times[1, n] \rightarrow S_{t^{\prime \prime}}$, there exists $t \geqq$ $\max \left\{t^{\prime}, t^{\prime \prime}\right\}, t<1$, so that $g^{\prime}\left(B^{3} \times\{i\}\right) \cap g^{\prime \prime}\left(B^{3} \times\{j\}\right) \cap S_{t}=\varnothing$ for any $i, j$. Thus the proof of Lemma 3.2 can be carried out using the 3-cells $g^{\prime}\left(B^{3} \times\{i\}\right) \cap S_{t}(i=1, \ldots, m)$ and $g^{\prime \prime}\left(B^{3} \times\{j\}\right) \cap S_{t}(j=1, \ldots, n)$ in the construction of $g$.

Proof of (3.1). We are going to construct a homeomorphism $H$ of $\boldsymbol{R}^{4}$ that carries $\psi[0,1]$ onto a locally flat arc. Since, in effect, $\psi[0,1]$ is an arbitrary subinterval in $\psi(\boldsymbol{R})$, this will prove that $\psi(\boldsymbol{R})$ is locally flat.

Let $\varepsilon_{1}, \varepsilon_{2}, \ldots$ be a sequence of positive numbers such that $\sum_{i=1}^{\infty} \varepsilon_{i}<\infty$.

Inductively we can use (3.2) and (3.3) to find numbers $0 \leqq t_{1}^{\prime}<t_{2}^{\prime}<\cdots<1$ and embeddings $g_{k}^{\prime}: B^{3} \times\left[-1,2^{k}+1\right] \rightarrow S_{t_{k}^{\prime}}$ such that $g_{k}^{\prime}(0, i)=\psi\left(i / 2^{k}\right)$ and

$$
g_{k+1}^{\prime}\left(B^{3} \times\{2 i\}\right)=g_{k}^{\prime}\left(B^{3} \times\{i\}\right) \cap S_{t_{k+1}^{\prime}} .
$$

Let $g_{1}, g_{2}, \ldots$ be a subsequence of $g_{1}^{\prime}, g_{2}^{\prime}, \ldots$ (with corresponding subsequences $t_{1}, t_{2}, \ldots$ of $t_{1}^{\prime}, t_{2}^{\prime}, \ldots$ and $n_{1}, n_{2}, \ldots$ of $\left.2^{1}+1,2^{2}+1, \ldots\right)$ chosen so that

$$
\operatorname{diam} g_{i}\left(B^{3} \times[j, j+1]\right)<\varepsilon_{i} \quad\left(-1 \leqq j<n_{i}\right) .
$$

Set $J_{i}=g_{i}\left(\{0\} \times\left[0, n_{i}-1\right]\right), B_{i j}=g_{i}\left(B^{3} \times[j, j+1]\right)$, and $B_{i}=\bigcup_{j=-1}^{n_{i}-1} B_{i j}$. Observe that whenever $k>i$ and $0 \leqq j \leqq n_{i}-2, J_{i} \cap B_{i j}, J_{k} \cap B_{i j}$, and $\psi([0,1]) \cap B_{i j}$ are properly embedded arcs in $B_{i j}$ sharing common endpoints. Let $h_{i}^{\prime \prime}: J_{i} \rightarrow \psi([0,1])$ be a homeomorphism taking $J_{i} \cap B_{i j}$ onto $\psi([0,1]) \cap B_{i j}$ that fixes the endpoints of $J_{i} \cap B_{i j}$, and let $h_{i}^{\prime}$ be a homeomorphism of $\boldsymbol{R}^{4}$ such that $h_{i}^{\prime} \mid \operatorname{Bd} B_{i j}$ and $h_{i}^{\prime} \mid \boldsymbol{R}^{4}-B_{i}$ are the identity and $h_{i}^{\prime} \mid J_{i}=\left(h_{i+1}^{\prime \prime}\right)^{-1} h_{i}^{\prime \prime}$. Thus $h_{i}^{\prime}$ is an $\varepsilon_{i}$-homeomorphism and $h_{i}^{\prime}\left(J_{i}\right)$ $=J_{i+1}$.

Observe also that if $U$ is any neighborhood of $J_{i}(i \geqq 2)$, then there exists an $\varepsilon_{i}$-homeomorphism $\theta_{i}$ of $\boldsymbol{R}^{4}$ such that $\theta_{i} \mid\left(\boldsymbol{R}^{4}-B_{i-1}\right) \cup J_{i}=$ identity and $\theta_{i}(U) \supset B_{i}$.

For $0<r \leqq 1$ let $B_{r}^{3}=\left\{r x \mid x \in B^{3}\right\}$ and let $C_{i}=g_{1}\left(B_{1 / i}^{3} \times\left[-1 / i, n_{1}-1+1 / i\right]\right)$.

Let $h_{1}=h_{1}^{\prime}$. Assuming $h_{k}: \boldsymbol{R}^{4} \rightarrow \boldsymbol{R}^{4}$ is defined such that $h_{k}\left(J_{1}\right)=J_{k+1}$, let $\theta_{k+1}$ be an $\varepsilon_{k+1}$-homeomorphism of $\boldsymbol{R}^{4}$ such that $\theta_{k+1} \mid\left(\boldsymbol{R}^{4}-B_{k}\right) \cup J_{k+1}=$ identity and $\theta_{k+1} h_{k}\left(C_{k+1}\right) \supset B_{k+1}$, and let $h_{k+1}=h_{k+1}^{\prime} \theta_{k+1} h_{k}$. Then $h_{k+1}$ moves no point of $\boldsymbol{R}^{4}$ farther than $2 \sum_{i=1}^{k+1} \varepsilon_{i}$. Thus the sequence $h_{1}, h_{2}, \ldots$ converges to a map $h$ of $\boldsymbol{R}^{4}$ onto itself. By construction $h\left|J_{1}=\lim h_{i}\right| J_{1}=h_{1}^{\prime \prime}: J_{1} \rightarrow \psi([0,1])$ is a homeomorphism and $h \mid R^{4}-B_{1}=$ identity. Let $p$ be a point of $B_{1}-J_{1}$. There exists $i$ such that $p \in B_{1}-C_{i}$. Thus $h_{i+1}(p) \notin B_{i+1}$ and so $h(p)=h_{i+2}(p)$. This implies that $h \mid B_{1}-J_{1}$ is a homeomorphism of $B_{1}-J_{1}$ onto $B_{1}-\psi([0,1])$, and hence $h$ is a homeomorphism. The homeomorphism $H=h^{-1}$ takes $\psi([0,1])$ onto an arc $\left(J_{1}\right)$ that is known to be locally flat. Hence $\psi([0,1])$ is locally flat. 
4. Proof of the main theorem. In the following, $K^{0}$ denotes the set of vertices of the complex $K$.

THEOREM 4.1. Let $K$ be a finite 1-complex topologically embedded in the interior of the PL 4-manifold $M^{4}$. Suppose that $K-K^{0}$ has mapping cylinder neighborhoods in $M^{4}$ at each point. Then $K$ is tame in $M^{4}$.

Proof. For each $x \in K-K^{0}$, let $V_{x}$ be an open arc in $K-K^{0}$ containing $x$ such that there exist an open 3-manifold $U_{x}$, a proper map $\phi_{x}$ of $U_{x}$ onto $V_{x}$, and an embedding $\psi_{x}$ of $Z_{\phi_{x}}$ into $M$ such that $\psi_{x}(v)=v$ for all $v \in V_{x}$. Applying (1.2), we see that $\phi_{x}^{-1}(t)$ has property $\mathrm{uv}^{1}(Z)$ for each $t \in V_{x}$. Applying (2.1), we see that there is a locally finite subset $F_{x}$ of $V_{x}$ such that if $J$ is an open interval in $V_{x}-F_{x}$ then $\phi_{x}^{-1}(J) \approx S^{2} \times \boldsymbol{R}$. By shrinking the interval $V_{x}$ somewhat, we may assume that $F_{x}$ is actually finite.

Now, let $\left\{V_{x_{1}}, V_{x_{2}}, \ldots\right\}$ be a locally finite subcollection of $\left\{V_{x}\right\}$ which covers $K-K^{0}$, and let $F=K^{0} \cup \bigcup_{i=1}^{\infty} F_{x_{i}} . F$ is a compact countable subset of $K$. The crucial property that $F$ enjoys is the following: For each point $x \in K-F, V_{x}, U_{x}, \phi_{x}, \psi_{x}$ may be chosen so that $\phi_{x}^{-1}(J) \approx S^{2} \times \boldsymbol{R}$ for any open interval $J \subset V_{x}$. By the special case (3.1), $\psi_{x}\left(V_{x}\right)$ is locally flat in $M^{4}$. Hence, $K$ is locally tame in $M^{4}$ at each point of $K-F$. It follows from [5] and [8] that $K$ is tame in $M^{4}$.

COROLlaRY 4.2. If $S$ is a 1-sphere in $S^{4}$ which has mapping cylinder neighborhoods in $S^{4}$ at every point, then $S$ is flat.

\section{REFERENCES}

1. S. Armentrout and T. M. Price, Decompositions into compact sets with UV properties, Trans. Amer. Math. Soc. 141 (1969), 433-442. MR 39 \#6307.

2. M. Brown, A proof of the generalized Schoenflies theorem, Bull. Amer. Math. Soc. 66 (1960), 74-76. MR 22 \#8470b.

3. J. L. Bryant, On embeddings of compacta in Euclidean space, Proc. Amer. Math. Soc. 23 (1969), 46-51. MR 39 \#6286.

4. J. L. Bryant and D. W. Sumners, On embeddings of 1-dimensional compacta in a hyperplane in $E^{4}$, Pacific J. Math. 33 (1970), 555-557.

5. J. C. Cantrell, $n$-frames in euclidean $k$-space, Proc. Amer. Math. Soc. 15 (1964), 574-578. MR 29 \#1627.

6. A. V. Cernavskií, Topological embeddings of manifolds, Dokl. Akad. Nauk SSSR 187 (1969), 1247-1250= Soviet Math. Dokl. 10 (1969), 1037-1041.

7. L. C. Glaser, "On double suspensions of arbitrary nonsimply connected homology $n$-spheres," Topology of manifolds, J. C. Cantrell and C. H. Edwards, Jr. (editors), Markham, Chicago, 1970.

8. H. Gluck, Embeddings in the trivial range, Ann. of Math. (2) 81 (1965), 195-210. MR 30 \#3456.

9. L. S. Husch and T. M. Price, Finding a boundary for a 3-manifold, Ann. of Math. (2) 91 (1970), 223-235.

10. R. C. Kirby, On the set of non-locally flat points of a submanifold of codimension one, Ann. of Math. (2) 88 (1968), 281-290. MR 38 \#5193. 
11. H. Kneser, Geschlossene Flächen in dreidimensionalen Mannigfaltigkeiten, Jber. Deutsch. Math.-Verein 38 (1929), 248-260.

12. R. C. Lacher, Cell-like mappings. I, Pacific J. Math. 30 (1969), 717-731. MR 40 \#4941. 13. Cell-like mappings. II, Pacific J. Math. 35 (1970), 649-660.

14. R. C. Lacher and A. H. Wright, "Mapping cylinders and 4-manifolds," Topology of manifolds, J. C. Cantrell and C. H. Edwards, Jr. (editors), Markham, Chicago, 1970.

15. D. R. McMillan, Jr., Acyclicity in three-manifolds, Bull. Amer. Math. Soc. 76 (1970), 942-964.

16. — UV properties and related topics, Lecture Notes, Florida State University, Tallahassee, Fla., 1970.

17. V. Nicholson, Mapping cylinder neighborhoods, Trans. Amer. Math. Soc. 143 (1969) 259-268. MR 40 \#2038.

18. A. H. Wright, Mappings from 3-manifolds onto 3-manifolds (to appear).

Department of Mathematics, Florida State University, Tallahassee, Florida 32306 